\title{
CORRIGENDUM
}

\section{Finite-size evaporating droplets in weakly compressible homogeneous shear turbulence - CORRIGENDUM}

\author{
Nicolò Scapin, Federico Dalla Barba, Giandomenico Lupo, \\ Marco Edoardo Rosti, Christophe Duwig and Luca Brandt
}

doi:10.1017/jfm.2021.1140, Published by Cambridge University Press, 14 January 2022

The authors apologise that upon publication of this article one of the authors names was spelled incorrectly. The correct spelling should be: Marco Edoardo Rosti

The online version of this article has been updated.

\section{REFERENCE}

Scapin, N., Dalla Barba, F., Lupo, G., Rosti, M., Duwig, C. \& Brandt, L. 2022 Finite-size evaporating droplets in weakly compressible homogeneous shear turbulence. J. Fluid Mech. 934, A15. doi:10.1017/jfm.2021.1140 